\title{
Effects of CMC Molar Mass on Mechanical Properties of CMC-Acid Gel
}

\author{
Machiko Takigami, Akihiro Hiroki", Naotsugu Nagasawa*, Takamitsu Kasahara ${ }^{* *}$, \\ Shoji Takigami** and Masao Tamada \\ Gunma Industry Support Organization, c/o Environmental Polymer Group, Japan Atomic Energy Agency, 1233 \\ Watanuki, Takasaki, Gunma 370-1292, Japan \\ Fax: 81-277-346-9694, e-mail:takigami.machiko@jaea.go.jp \\ *Environmental Polymer Group, Japan Atomic Energy Agency, 1233 Watanuki, Takasaki, Gunma 370-1292, \\ Japan \\ Center for Material Research by Instrumental Analysis, Gunma University, 1-5-1 Tenjincho, Gunma 376-8515, \\ Japan
}

\begin{abstract}
Carboxymethyl cellulose (CMC) with different molar mass was prepared by acid hydrolysis at $121^{\circ} \mathrm{C}$. CMC thus prepared was mixed with citric acid aqueous solution to form CMC-acid gel. By replacing sodium existing as counter ion in carboxymethyl group with hydrogen, $\mathrm{CMC}$ molecules coagulate and hydrogen bonds are formed among $\mathrm{CMC}$ molecules. The $\mathrm{CMC}$-acid gel prepared in that way was subjected to evaluation of gel fraction, water absorption and mechanical properties. Gel fraction was higher in $\mathrm{CMC}$ with higher molar mass than that in CMC with lower molar mass at short incubation time. However, there was no difference in gel fraction attributable to molar mass of $\mathrm{CMC}$ after long time incubation. CMC with higher molar mass crosslinked more easily than $\mathrm{CMC}$ with lower molar mass. The gel made of $\mathrm{CMC}$ with lower molar mass was softer and absorbed more amount of water than that with higher molar mass. All the results were elucidated by number of hydrogen bonds in CMC molecule. The gel made of higher molar mass CMC becomes stiff and brittle after long time incubation, however, the gel made of lower molar mass CMC keeps softness and strength longer.

Key words: CMC, molar mass, acid, gel, hydrogen bond
\end{abstract}

\section{INTRODUCTION}

Carboxymethyl cellulose (CMC) is a widely used water-soluble polymer derived from wood pulp. Because of its safe and environmentally friendly properties, its use extended after gelation by multivalent metal ions, crosslinking agents and radiation crosslinking. We found that $\mathrm{CMC}$ formed a gel (CMC-acid gel) by mixing with acid [1]. The mechanism of gel formation was elucidated as a replacement of sodium with hydrogen in carboxymethyl group existing as a counter ion. Commercial CMC is generally in the form of sodium salt owing to the production process. CMC in the form of sodium salt is easily soluble in water, however, it is not soluble in water once it is replaced with hydrogen. As the result, CMC molecules became close enough to form hydrogen bonds between the molecules and gel was formed in this way. The resultant gel is elastic, adherent and strong. Those characters were very specific as for polysaccharide gels.

We have examined the properties of CMC-acid gel consisting of $\mathrm{CMC}$ and Konjac glucomannan (KM), in which KM with different molar mass was used. The mechanical properties depended on the molar mass of KM [2]. When KM with higher molar mass was used, the gel was more elastic than that prepared by using KM with lower molar mass.

We have reported a method to decrease molar mass of polysaccharides using acid hydrolysis at elevated temperature and pressure [3]. By the method, KM with varieties of molar masses was prepared without chemical structural changes. Applying the method, CMC was hydrolyzed with citric acid to prepare $\mathrm{CMC}$ with various molar masses.

As we have not examined the effects of molar mass of CMC on mechanical properties of the CMC-acid gel in the former report [2], the effects are examined in the present work to seek 
application areas of CMC-acid gel.

\section{EXPERIMENTAL}

2.1 Materials

CMC 1380 having degree of substitution of 1.36 and viscosity of $1820 \mathrm{mPa} \cdot \mathrm{s}\left(1 \%\right.$ solution, $\left.25^{\circ} \mathrm{C}\right)$, produced by Daicel Chemical Industries, Ltd., Japan, was used throughout the experiment. It was in the form of sodium salt owing to its preparation process. Citric acid was reagent grade and used as received.

\subsection{Hydrolysis}

CMC was mixed with $0.05 \mathrm{M}$ citric acid solution at the concentration of $20 \%$. For mixing, a hybrid defoaming mixer (AR-250, Thinky Cooperation, Japan) was used. After mixing, the mixture in paste was transferred into an autoclavable polypropylene bag. Autoclaving was performed at $121^{\circ} \mathrm{C}$ for $1-30$ min using a table top autoclave (Pasolina IST-150, Chiyoda Seisakujo, Japan).

\subsection{Molar mass measurement}

Original and hydrolyzed $\mathrm{CMC}$ samples were dissolved in $50 \mathrm{mM}$ aqueous $\mathrm{NaNO}_{3}$ solution and filtered with $0.45 \mu \mathrm{m}$ cellulose acetate membrane filters (Millipore), and molar mass of the CMC was measured by multiangle static light-scattering (MALS) system equipped with a size exclusion chromatography (SEC) column of $\mathrm{GMPW}_{\mathrm{XL}}$ (Tosoh) as described in the former paper [3].

\subsection{FT-IR measurement}

FT-IR spectra of CMC before and after hydrolysis were measured by Magna $560+$ Continu $\mu$ MII (Nicolet, Thermo Fisher Scientific K.K.).

\subsection{Gelation of CMC}

Using the hybrid defoaming mixer, the autoclaved sample was mixed with citric acid solution. The final concentrations of CMC and 0.5 $M$ citric acid were $10 \%$ and $90 \%$, respectively.

The mixture was molded into a cylinder with a thickness of $10 \mathrm{~mm}$ and a diameter of $20 \mathrm{~mm}$. The cylinders were kept at 30 or $50^{\circ} \mathrm{C}$ to be served for the measurement of mechanical properties.

\subsection{Mechanical properties of CMC-acid gel}

Mechanical properties of the gels were measured using a table-top universal tester (EZ-Test, Shimadzu Co.). The CMC-acid gel cylinders were pressed at $2 \mathrm{~mm} / \mathrm{min}$.
2.7 Gel fraction and water absorption

After weighing the cylinders, they were immersed in purified water for 2 days to remove uncrosslinked CMC (soluble CMC) and acid. Then the samples were dried and weighed. The gel fraction and amount of water absorbed by the gel were defined as follows:

$$
\text { Gel fraction }(\%)=100 \times \mathrm{Wg} / \mathrm{Wi}
$$

where $\mathrm{Wi}$ and $\mathrm{Wg}$ are the weights of initial dry $\mathrm{CMC}$, and insoluble part after immersing in water, respectively.

$$
\text { Water absorbed by gel }=(\mathrm{Ws}-\mathrm{Wg}) / \mathrm{Wg}
$$

where Ws is the weight of swollen hydrogel.

\section{RESULTS AND DISCUSSION}

3.1 Effects of hydrolysis on molar mass and chemical structure of CMC

Molar mass of CMC hydrolyzed by $50 \mathrm{mM}$ citric acid at $121^{\circ} \mathrm{C}$ decreased with increase of autoclaving time as shown in Fig. 1.

The hydrolysis did not cause any big change in $\mathrm{CMC}$ as shown in Fig. 2. Carboxylmethyl group around $1620 \mathrm{~cm}^{-1}$ was the same in both CMC before and after hydrolysis with $50 \mathrm{mM}$ citric acid at $121^{\circ} \mathrm{C}$.

\subsection{Gel fraction}

The hydrolyzed $\mathrm{CMC}$ and $0.5 \mathrm{M}$ citric acid were mixed to prepare CMC-acid gel. Gel fraction was plotted against molar mass as shown in Fig. 3 and clearly showed that CMC with higher molar mass was crosslinked more easily than CMC with lower molar mass. The gel fraction increased with incubation time.

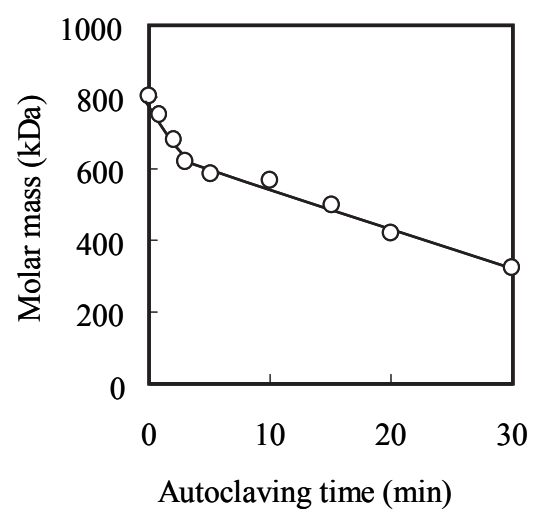

Fig. 1 Relation between autoclaving time and molar mass of CMC hydrolyzed by $50 \mathrm{mM}$ citric acid at $121^{\circ} \mathrm{C}$. 
$\mathrm{CMC}$-acid gel incubated at $50^{\circ} \mathrm{C}$ gave higher gel fraction than that incubated at $30^{\circ} \mathrm{C}$ at the initial stage of gelation, namely on the $4^{\text {th }}$ day of incubation. Incubation at higher temperature causes quicker hydrogen bond formation among CMC molecules.

\subsection{Water absorption by gel}

Water absorbed by gel increased with decrease of molar mass of CMC, and decreased with incubation time as shown in Fig. 4. Incubation at $50^{\circ} \mathrm{C}$ decreased water absorption because of the progress of hydrogen bonds formation among CMC molecules.

3.4 Mechanical properties of gel

Compression modulus of CMC-acid gel is shown in Fig. 5. Compression modulus decreased with decrease of molar mass meaning that gel became

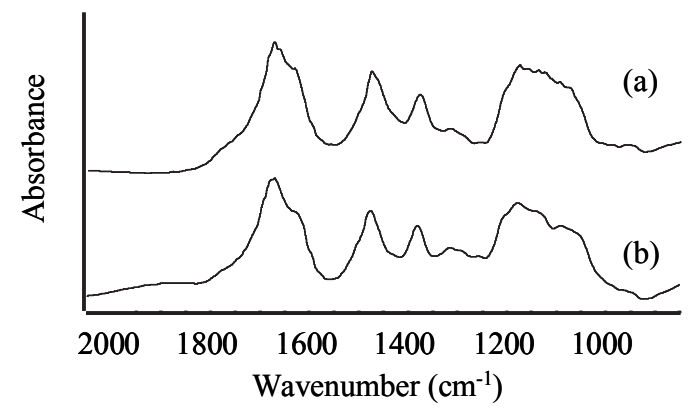

Fig. 2. FT-IR spectra of CMC before (a) and after (b) hydrolysis by $50 \mathrm{mM}$ citric acid for 10 $\min$ at $121^{\circ} \mathrm{C}$.

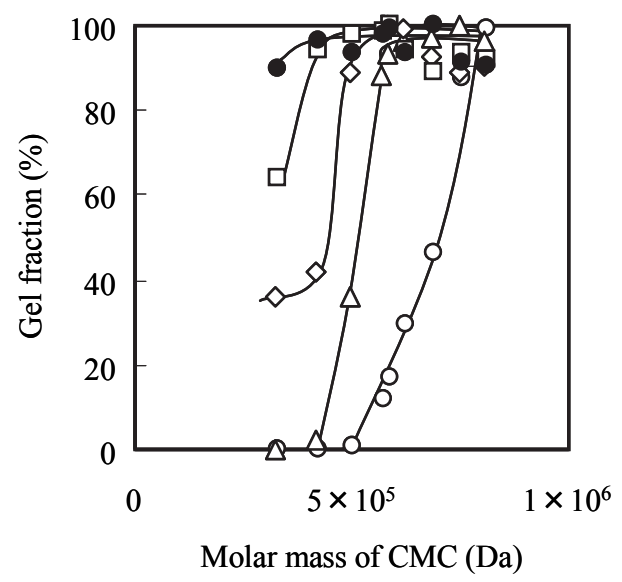

Fig. 3 Relation between molar mass of CMC and gel fraction. Gels were kept; $\bigcirc: 4$ days at $30^{\circ} \mathrm{C}$, $\triangle: 7$ days at $30^{\circ} \mathrm{C}, \diamond: 10$ days at $30^{\circ} \mathrm{C}, \square: 14$ days at $30^{\circ} \mathrm{C}, 0: 4$ days at $50^{\circ} \mathrm{C}$. softer. The high value in compression modulus using CMC with smaller molar mass less than 500,000 Da reflects hard sample stage of the universal tester.

All gels incubated at $30^{\circ} \mathrm{C}$ were not broken after applying a load of $4.8 \mathrm{~N} / \mathrm{mm}^{2}$. Gels with less gel fraction $(\sim 80 \%)$ enlarged and did not recover the initial thickness after compression, however, the gels with higher gel fraction $(80 \% \sim)$ recovered the thickness after compression as shown in Fig. 6. Elastic and strong gels were formed.

Some of the gels incubated at $50^{\circ} \mathrm{C}$ broke after incubation as shown in Fig. 7. At $50^{\circ} \mathrm{C}$, gelation proceeds rapidly compared to that at $30{ }^{\circ} \mathrm{C}$. Hydrogen bonds are formed among CMC molecules and the gels become brittle, however, gels made of smaller molar mass CMC keep elasticity longer.

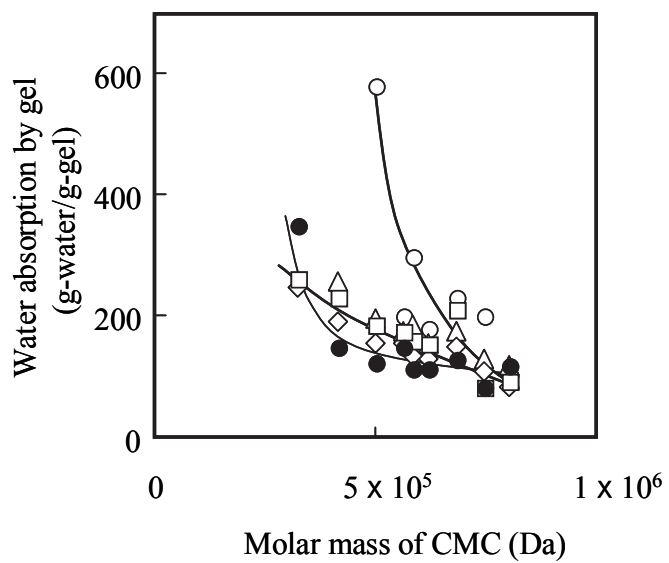

Fig. 4 Relation between molar mass of CMC and water absorption by gel. Notations are the same with those shown in Fig. 3.

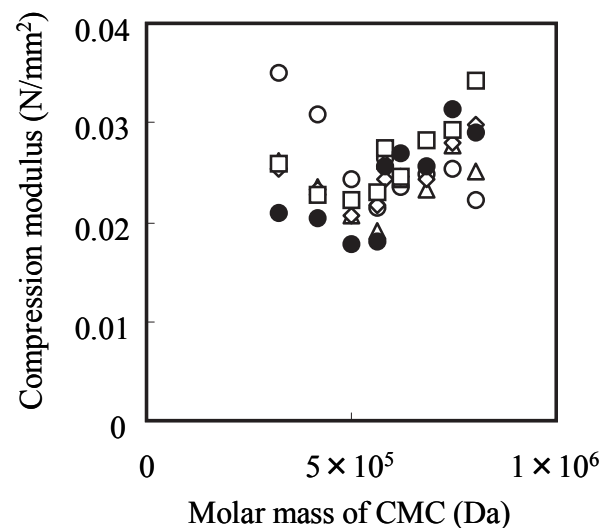

Fig. 5 Relation between molar mass of CMC and compression modulus. Notations are the same with those shown in Fig. 3. 


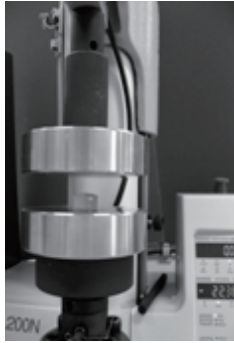

(A)

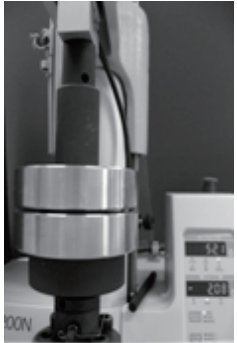

(B)

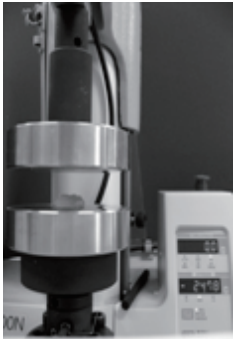

(C)

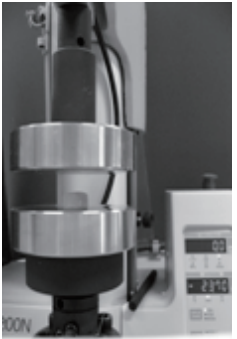

(D)
Fig. 6 CMC-acid gel under compression test.

(A): before compression

(B): under compression

(C): immediately after compression

(D): after removing load for $3 \mathrm{~h}$

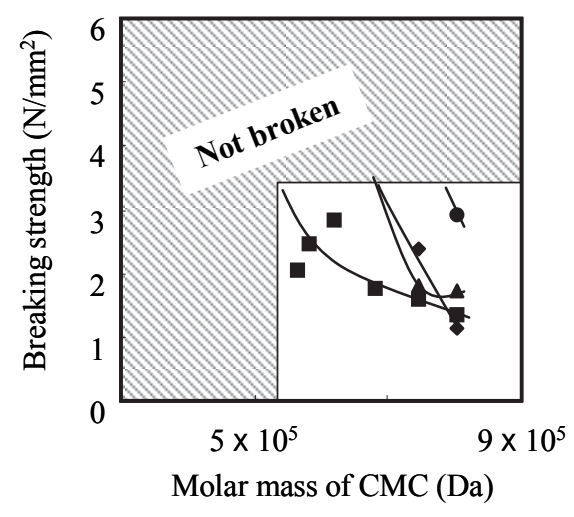

Fig. 7 Relation between molar mass of CMC and breaking strength. Gels were kept; $\bigcirc: 4$ days at $50^{\circ} \mathrm{C}, \boldsymbol{\Delta}: 7$ days at $50^{\circ} \mathrm{C}, \bullet: 10$ days at $50^{\circ} \mathrm{C}$, 14 days at $50^{\circ} \mathrm{C}$.

\subsection{Schematic model of CMC-acid gel}

CMC-acid gel composed of higher molar mass $\mathrm{CMC}$ has more crosslinking point in one CMC molecule than that composed of lower molar mass $\mathrm{CMC}$, as shown in Fig. 8. With the increase of incubation time and rise of the incubation temperature, the number of hydrogen bonds in CMC molecule increases and CMC-acid gel becomes stiff and fragile after incubation. When CMC with low molar mass was used for gel formation, hydrogen bonds formation proceeds gradually and gradually, keeping the $\mathrm{CMC}$-acid gel elastic and strong for a long time.

\section{Summary}

CMC-acid gels with different mechanical properties were prepared by using $\mathrm{CMC}$ with different molar mass. As CMC is an environmentally friendly polymer, applications of CMC-acid gels to some fields which require considerable strength and elasticity are suggested.

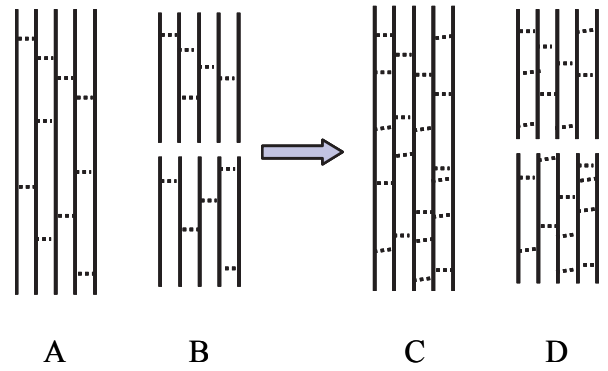

Fig. 8 Simplified schematic model of CMC-acid gel.

$\mathrm{A}$ and $\mathrm{C}$ : $\mathrm{CMC}$ with high molar mass, $\mathrm{B}$ and $\mathrm{D}$ : $\mathrm{CMC}$ with low molar mass. A and B: Initial stage of gelation, C and D: Latter stage of gelation.

Solid and dotted lines show CMC molecule and hydrogen bond, respectively.

Acknowledgment

The present study was carried out as a part of the project, "Collaboration of Regional Entities for the Advancement of Technological Excellence, Gunma", sponsored by Science and Technology Agency, Japan.

\section{References}

[1] M. Takigami, H. Amada, N. Nagasawa, T. Kasahara, S. Takigami and M. Tamada, Trans. Mat. Res. Soc. Jpn., 32[3], 713-716 (2007).

[2] T. Kasahara, M. Takigami, N. Nagasawa, M. Tamada and S. Takigami, Trans. Mat. Res. Soc. Jpn., 33[4], 927-930(2008).

[3] M. Takigami, P. Prawitwong, Y. Tahara, R. Ohtsuka, R. Takahashi and S. Takigami, Trans. Mat. Res. Soc. Jpn., 31[3], 723-726 (2006).

(Received December 23, 2008; Accepted April 16, 2009) 